\title{
Clinical significance of postoperative atrial arrhythmias in patients who underwent lung transplantation
}

\author{
Byung Gyu Kim ${ }^{1,}{ }^{\star} \dagger$, Jae-Sun Uhm ${ }^{2,}$, Pil-Sung Yang 3 , Hee Tae $\mathrm{Yu}^{2}$, Tae-Hoon Kim², Boyoung Joung ${ }^{2}$,
} Hui-Nam Pak ${ }^{2}$, Song Yee Kim ${ }^{4}$, Moo Suk Park ${ }^{4}$, Jin Gu Lee ${ }^{5}$, Hyo Chae Paik ${ }^{5}$, and Moon-Hyoung Lee ${ }^{2}$

\begin{abstract}
${ }^{1}$ Division of Cardiology, Department of Internal Medicine, Inje University Seoul Paik Hospital, Seoul; ${ }^{2}$ Division of Cardiology, Department of Internal Medicine, Severance Hospital, Yonsei University College of Medicine, Seoul; ${ }^{3}$ Division of Cardiology, Department of Internal Medicine, CHA Bundang Medical Center, CHA University, Seongnam; ${ }^{4}$ Division of Pulmonology, Department of Internal Medicine and ${ }^{5}$ Department of Thoracic and Cardiovascular Surgery, Severance Hospital, Yonsei University College of Medicine, Seoul, Korea
\end{abstract}

Received : September 7, 2018 Revised : December 11, 2018 Accepted: January 18, 2019

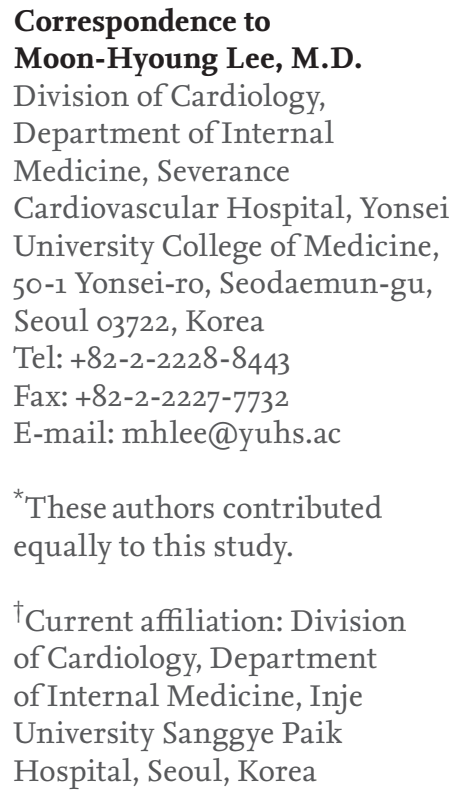

Background/Aims: Atrial arrhythmia (AA) occasionally occurs after lung transplantation (LT); however, risk factors for AA and their impact on clinical outcomes are inconsistent. We aimed to investigate the incidence, predisposing factors, and clinical outcomes of AA after LT.

Methods: We retrospectively evaluated 153 consecutive patients who underwent LT between January 2010 and August 2016. An AA episode was defined as a documented atrial fibrillation (AF), atrial flutter, or atrial tachycardia on 12-lead electrocardiography or episodes lasting $\geq 30$ seconds on telemetry monitoring.

Results: The mean follow-up time was $22.0 \pm 19.1$ months. Postoperative AA occurred in 46 patients (30.1\%) after LT. Patients with postoperative AA were older, had larger body surface area, and had an increased incidence of paroxysmal AF prior to transplantation, idiopathic pulmonary fibrosis, and postoperative tracheostomy than patients without AA. Preoperative right atrial pressure (RAP) (odds ratio $[\mathrm{OR}], 1.19 ; p=0.005)$ and longer periods of mechanical ventilation $(\mathrm{OR}, 1.03$; $p=0.008)$ were found to be independent risk factors for AA after surgery. Development of AA was a significant predictor of long-term overall mortality (hazard ratio, 2.75; $p=0.017$ ).

Conclusions: Patients with elevated preoperative RAP and long-term ventilator care had a higher risk of AA after LT. Further, AA after LT was associated with poor long-term survival.

Keywords: Lung transplantation; Atrial fibrillation; Atrial flutter; Tachycardia, ectopic atrial; Survival

\section{INTRODUCTION}

Lung transplantation (LT) has been performed increasingly over the past 2 decades [1,2]. Atrial arrhythmias (AA), including atrial fibrillation $(\mathrm{AF})$, atrial flutter (AFL), and atrial tachycardia (AT), are highly prevalent complications after
LT, with a reported incidence ranging from $25 \%$ to $45 \%$ depending on their definition and methods of detection [3-9]. At present, there is inconsistent evidence supporting the association between mortality after LT and postoperative AA $[3,6,10,11]$. In addition, different risk factors have been reported 
with inconsistent results $[3,7,11]$. An increasing number of $\mathrm{LT}$ recipients at an advanced age, bilateral nature of LT, development of donor selection criteria, donor management protocols, and postoperative management are some current noteworthy changes, especially after the implementation of the lung allocation score in 2005 $[1,12]$, which necessitated re-evaluation of these arrhythmias. Therefore, the purpose of this study was to assess the incidence of AA in patients who underwent LT and to determine its risk factors and impact on clinical outcomes in the contemporary era.

\section{METHODS}

\section{Patient population}

This was a single-centered, retrospective cohort analysis. The study was approved by the Institutional Review Board of Severance Hospital (IRB number: 2013-0522035) and was conducted in compliance with the Declaration of Helsinki. Informed consent and a critical event committee were exempted by the board due to this study's retrospective design. Lung transplant patients, who underwent either single or bilateral LT, between January 2010 and August 2016, were eligible for the study. Patients who underwent heart-lung transplantation or were lost to follow-up were excluded from this study.

\section{Data collection}

Baseline characteristics, operative and postoperative clinical variables, and clinical outcomes during the follow-up period were captured from electronic medical records. Baseline demographics collected included: age, gender, comorbidities, history of AF, preoperative medications, indications for LT, electrocardiography (ECG), echocardiographic data, right heart catheterization data (if available), and laboratory results. Operation notes were reviewed to obtain details of the surgery such as transplant type (single or bilateral), surgical procedure, and intraoperative complications. Preoperative echocardiographic data included left ventricular ejection fraction (LVEF), size of the cardiac chambers, right atrial pressure (RAP), right ventricular systolic pressure, and E/E' ratio. LVEF and left atrial volume were determined using the biplane Simpson's method. RAP was estimat- ed by evaluating the inferior vena cava during respiration. Right ventricular systolic pressure was calculated by adding the estimated RAP to the pressure gradient between the right atrium and right ventricle $(4 \times$ peak tricuspid regurgitant jet velocity ${ }^{2}$ ). Further, to determine if postoperative clinical features predicted postoperative AA, data on the duration of mechanical ventilation needed and cardiac markers were collected as well.

\section{Study outcomes}

All patients completed continuous ECG monitoring in the surgical intensive care unit (ICU). The outcomes collected included development of AA (including early and late postoperative AA), postoperative length of ICU stay, length of hospital stay, postoperative occurrences of stroke, and all-cause mortality. Early postoperative AA was defined as the first documentation of AF, AFL, or AT lasting $\geq 30$ seconds via either a 12-lead ECG or rhythm strips obtained from the ECG monitoring within 30 days of transplantation. Late AA was defined as the occurrence of AA, after 30 days of transplantation, at any time during the follow-up period. ECGs were analyzed by two independent cardiologists (B.G.K. and J.S.U.). Progress notes, emergency room records, and consultation notes were also intensively reviewed to determine the physician's assessment of arrhythmia or tachycardia. Onset time of postoperative AA was defined as the number of days from the date of LT to the date of the first episode of AA. Lengths of ICU stay and hospital stay were calculated from the date of LT to the date of transfer to the general ward and discharge, respectively. Postoperative occurrence of stroke was defined as occurrence of a focal neurological deficit confirmed from abnormal findings of brain imaging studies by a neurologist after surgery. All-cause mortality included any death after LT. Treatment strategies for AA episodes were stratified into rate control ( $\beta$-blockers, non-dihydropyridine calcium channel blockers, and digoxin) and rhythm control (anti-arrhythmic drugs and electrical cardioversion). Decisions regarding treatments were at the discretion of the transplant surgeons or consulting cardiologists.

\section{Statistical analysis}

Continuous variables were presented as mean \pm standard deviation and categorical variables were presented as number and percentage. Mean differences between 


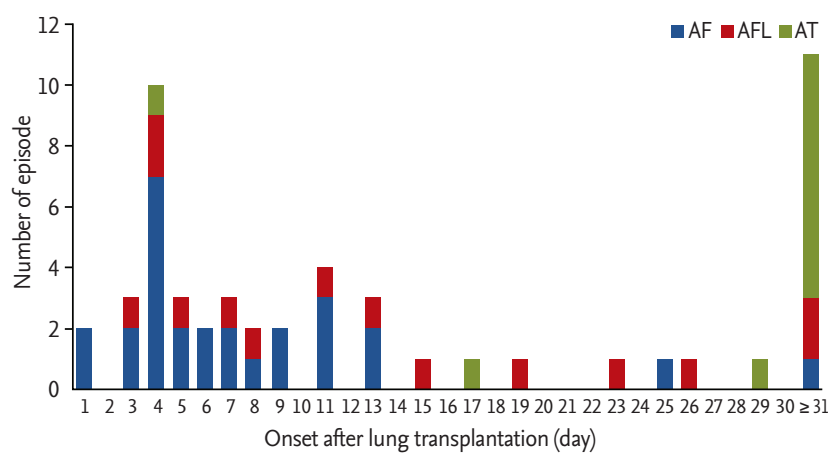

Figure 1. Incidence and time to onset of atrial arrhythmias after lung transplantation. AF, atrial fibrillation; AFL, atrial flutter; AT, atrial tachycardia.

groups were tested using the Student's $t$ test for normally distributed data or the Mann-Whitney $U$ test for skewed data. Categorical variables were analyzed using the Pearson's chi-square test or Fisher's exact test. Uni- and multivariate analyses using logistic regression models were constructed to validate predictors of AA. Variables with $p<0.1$ from the univariate analyses were considered as potential risk factors of postoperative AA in the multivariate analyses. Log-rank and Kaplan-Meier tests were used to compare survival between groups. Multivariate analysis using Cox proportional hazards models was performed to identify whether postoperative AA development was one of the independent risk factors for mortality. All statistical analyses were carried out in SPSS version 23.0 (IBM Corp., Armonk, NY, USA). All tests were two-sided, and the results were considered statistically significant at $p<0.05$.

\section{RESULTS}

\section{Patient demographics}

In total, 153 patients (age, $51.2 \pm 13.6$ years; men, 58.8\%) who underwent LT were evaluated. Bilateral and single LT was performed in 141 (92.2\%) and 12 (7.8\%) patients, respectively. The major indications for LT were (in order of frequency) idiopathic pulmonary fibrosis (IPF), graft-versus-host disease after hematopoietic stem cell transplantation, lymphangioleiomyomatosis, bronchiectasis, and chronic obstructive pulmonary disease. Less frequent diagnoses included idiopathic pulmonary hypertension, miliary tuberculosis, pulmonary capillary hemangioma, Langerhans cell histiocytosis, and interstitial lung diseases associated with systemic rheumatic diseases. Comorbidities of the patients included hypertension (20.9\%), diabetes (17.6\%), dyslipidemia (9.8\%), and coronary artery disease (7.2\%). All patients had sinus rhythm at the time of surgery, but five patients (3.3\%) had a history of paroxysmal AF prior to the transplant. All patients were treated with standard immunosuppressive medications, such as tacrolimus, azathioprine, and prednisolone, after surgery.

\section{Incidence, timing, and types of AA}

The mean follow-up period post-transplantation was $22.0 \pm 19.1$ months. During the follow-up period, AA developed in 46 of the 153 of patients (30.1\%). Early postoperative AA occurred in 40 patients $(26.1 \%)$ with AA, of whom 26 (17.0\%) had AF, 11 (7.2\%) had AFL, and three (2.0\%) had AT. There were no significant differences in the onset time among patients with AF, AFL, and AT (7.2 $\pm 5.3,11.3 \pm 6.9$, and 17.3 \pm 10.3 days, respectively; $p=0.195$ ). The occurrence of early postoperative AA peaked on the postoperative day (POD) 4 . In $67.5 \%$ of patients, AA developed within $\mathrm{POD} 10$, and in $90.0 \%$ of patients before POD 20 (Fig. 1). The conversion of AA into sinus rhythm was observed in all patients during hospitalization and the mean duration of early postoperative AA was $2.6 \pm$ 2.6 days (range, 1 to 11 ). Late AA occurred in 11 patients (7.2\%), of whom one (0.7\%) had AF, two (1.3\%) had AFL, and eight $(5.2 \%)$ had AT. The median interval from LT to onset of late AA was 64 days (interquartile range, 45 to 95). AF and AT were the most common AA in early and late postoperative periods $(65.0 \%$ and $72.7 \%$, respectively) (Fig. 1). Conversion of late AA into sinus rhythm was also observed in all patients with a duration of 8.2 \pm 10.4 days (range, 1 to 32 ). Five patients developed both early postoperative and late AA: three patients with early $\mathrm{AF}$ and one patient with early AFL developed AT: one patient with early AF developed AFL in the late postoperative period (Fig. 2) [13].

\section{Risk factors for AA}

Differences in patients' characteristics according to the development of AA are presented in Table 1. Patients with AA were significantly older, had larger body surface area (BSA), and had frequent incidences of AF before transplantion, IPF, and postoperative tracheostomy 


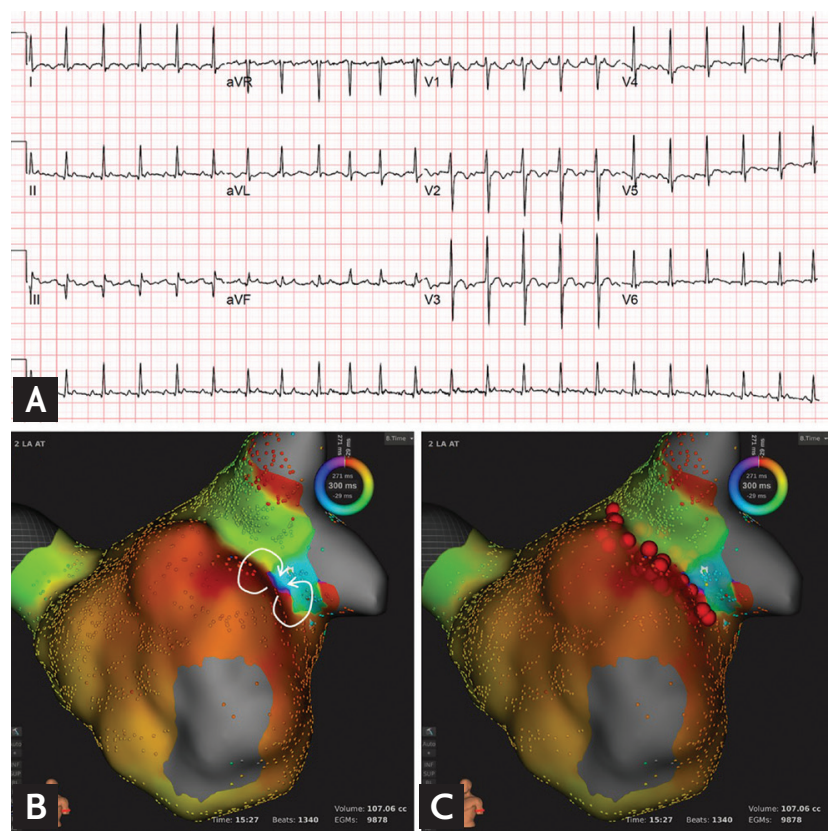

Figure 2. A case of a 62-year-old man who was treated for atrial tachycardia with radiofrequency catheter ablation. (A) Electrocardiography showing atrial tachycardia. (B) Three-dimensional activation map reveals figure-of-eight intra-atrial reentrant tachycardia (white arrows) with the slow conduction zone at the anastomosis line. (C) Radiofrequency catheter ablation was performed along the ridge (red balls). Adaptad from Uhm et al. [13]. aVR, augmented Vector right; aVL, augmented Vector left; aVF, augmented Vector foot.

than those without AA. No significant differences were observed between patients with and those without AA in preoperative echocardiographic data (including LVEF, left atrial size, RAP, right ventricular systolic pressure, and $\left.E / E^{\prime}\right)$, and cardiac catheterization data (including mean pulmonary artery pressure, mean right atrial pressure, and mean pulmonary capillary wedge pressure). Predictors of AA at any time after LT are shown in Table 2. Longer periods of mechanical ventilation (hazard ratio [HR], 1.03; 95\% confidence interval [CI], 1.01 to 1.06; $p$ $=0.008$ ) and preoperative RAP (HR, 1.19; 95\% CI, 1.06 to 1.35; $p=0.005$ ) were found to be significant risk factors for AA after LT, when adjusted for other risk factors.

\section{Treatment strategies for AA}

Forty of the 46 patients $(87.0 \%)$ received treatment for AA. The remaining six patients, with early postoperative AA (three AF, one AFL, and two AT), were not treated as their heart rates remained stable and the AA was spon- taneously converted to sinus rhythm within $1.8 \pm 1.5$ days. Rate control treatment, using $\beta$-blockers, calcium channel blockers (diltiazem or verapamil), and digoxin, was used in the majority of patients (39 of 40, 97.5\%). Six of the 40 patients $(16.7 \%)$ were treated with combined antiarrhythmic medical therapy (amiodarone), and two patients underwent electrical cardioversion due to hemodynamic instability. Non-vitamin K-dependent oral anticoagulants were administered to five patients with an AF lasting for $>2$ days and a $\mathrm{CHA}_{2} \mathrm{DS}_{2}-\mathrm{VASc}$ score $\geq 2$.

\section{Clinical impact of AA}

Length of ICU stay and hospital stay, as well as in-hospital mortality, were not significantly different between patients with and without AA (Table 1). Stroke developed in six patients (3.9\%) after LT, and the incidence was similar between patients with and without AA. Long-term overall mortality was significantly higher in patients who developed AA in comparison to their counterparts (Fig. 3). Landmark analyses were performed to evaluate survival 30 days after LT. Incidence of all-cause mortality was higher in the patients with early postoperative AA than in those without AA (Fig. 4). Further, patients who developed late AA or both early and late AA had greater rate of all-cause mortality than those without AA. (Fig. 4).

The major causes of death in all LT recipients included infection (63.0\%) and multi-organ failure syndrome (16.1\%). There was no difference in causes of death between the patients with and without AA (infection: 64.0\% vs. $62.1 \%, p=0.872$; multi-organ failure syndrome: $16.0 \%$ vs. $13.8 \%, p=0.826$ ). All-cause mortality and cause of death were summarized in Table 3 .

Postoperative AA, including early and late AA, was found to be an independent predictor of overall mortality, even after adjustment for other covariates. An E/E' $>15$ and longer periods of mechanical ventilation were also identified as independent risk factors associated with increased mortality after LT (Table 4).

\section{DISCUSSION}

The major findings of this study were as follows: (1) AA was common after LT; (2) elevated preoperative RAP and long-term ventilator care were independent predictors for the development of AA after LT; and (3) all-cause 
Table 1. Baseline characteristics of patients with and without postoperative atrial arrhythmia

\begin{tabular}{|c|c|c|c|}
\hline Characteristic & $\mathrm{AA}(\mathrm{n}=46)$ & No AA $(n=107)$ & $p$ value \\
\hline Age at transplant, yr & $57.0 \pm 10.7$ & $48.8 \pm 14.0$ & $<0.001$ \\
\hline Male sex & $32(69.6)$ & $58(54.2)$ & 0.077 \\
\hline Body surface area, $\mathrm{m}^{2}$ & $1.65 \pm 0.19$ & $1.56 \pm 0.24$ & 0.042 \\
\hline Body mass index, $\mathrm{kg} / \mathrm{m}^{2}$ & $21.7 \pm 4 \cdot 3$ & $20.9 \pm 3.7$ & 0.275 \\
\hline \multicolumn{4}{|l|}{ Comorbidities } \\
\hline Hypertension & $12(26.1)$ & $20(18.7)$ & 0.302 \\
\hline Diabetes & $12(26.1)$ & $15(14.0)$ & 0.073 \\
\hline Dyslipidemia & $7(15.2)$ & $8(7.5)$ & 0.149 \\
\hline Smoking & $22(47.9)$ & $41(38.3)$ & 0.273 \\
\hline Coronary artery disease & $6(13.0)$ & $5(4 \cdot 7)$ & 0.088 \\
\hline Stroke & $1(2.2)$ & $1(0.9)$ & 0.512 \\
\hline Atrial fibrillation & $4(8.7)$ & $1(0.9)$ & 0.029 \\
\hline Serum creatinine, $\mathrm{mg} / \mathrm{dL}$ & $0.7 \pm 0.3$ & $0.7 \pm 0.4$ & 0.336 \\
\hline \multicolumn{4}{|l|}{ Indication for transplant } \\
\hline Idiopathic pulmonary fibrosis & $34(73.9)$ & $56(52.8)$ & 0.015 \\
\hline GVHD & $3(6.5)$ & $10(9.4)$ & 0.755 \\
\hline COPD & $3(6.5)$ & $4(3.8)$ & 0.433 \\
\hline Bronchiectasis & $2(4 \cdot 3)$ & $5(4 \cdot 7)$ & 0.921 \\
\hline Lymphangioleiomyomatosis & $1(2.2)$ & $8(7.5)$ & 0.278 \\
\hline Other & $3(6.5)$ & $23(21.5)$ & 0.024 \\
\hline \multicolumn{4}{|l|}{ Type of lung transplantation } \\
\hline Bilateral & $43(93.5)$ & $98(91.6)$ & 0.487 \\
\hline \multicolumn{4}{|l|}{ Echocardiography findings } \\
\hline Ejection fraction, \% & $64.3 \pm 9.8$ & $64.3 \pm 11.4$ & 0.981 \\
\hline Left atrial volume index, $\mathrm{mL} / \mathrm{m}^{2}$ & $19.1 \pm 7.2$ & $18.0 \pm 7.2$ & 0.596 \\
\hline $\mathrm{RAP}, \mathrm{mmHg}$ & $8.7 \pm 5.2$ & $7.2 \pm 3.9$ & 0.075 \\
\hline RVSP, mmHg & $53.6 \pm 27.6$ & $49.2 \pm 21.6$ & 0.332 \\
\hline $\mathrm{E} / \mathrm{E}^{\prime}$ & $10.6 \pm 4.5$ & $10.5 \pm 4.1$ & 0.889 \\
\hline \multicolumn{4}{|l|}{ Right-sided heart catheterization data } \\
\hline Mean PAP, mmHg & $29.1 \pm 14.4$ & $28.2 \pm 10.4$ & 0.752 \\
\hline Mean RAP, mmHg & $6.8 \pm 3.7$ & $8.0 \pm 5.0$ & 0.275 \\
\hline Mean PCWP, mmHg & $10.8 \pm 5.3$ & $11.2 \pm 6.7$ & 0.756 \\
\hline \multicolumn{4}{|l|}{ Pre- and postoperative data } \\
\hline Preoperative PCPS & $10(22.2)$ & $22(20.8)$ & 0.840 \\
\hline Preoperative MV & $16(35.6)$ & $30(28.3)$ & 0.376 \\
\hline Postoperative TnT & $0.810 \pm 0.891$ & $0.491 \pm 0.264$ & 0.056 \\
\hline Postoperative tracheostomy & $35(76.1)$ & $45(42.5)$ & $<0.001$ \\
\hline Perioperative MV duration, day & $27.2 \pm 20.5$ & $17.8 \pm 21.8$ & 0.020 \\
\hline Length of postoperative ICU stay, day & $14.5 \pm 10.0$ & $13.1 \pm 12.1$ & 0.541 \\
\hline Length of hospital stay, day & $63.5 \pm 55.1$ & $47.8 \pm 37.3$ & 0.085 \\
\hline In-hospital mortality & $4(8.7)$ & $5(4.7)$ & 0.242 \\
\hline New onset of stroke & $3(6.5)$ & $3(2.8)$ & 0.360 \\
\hline
\end{tabular}

Values are presented as mean $\pm \mathrm{SD}$ or number (\%).

AA, atrial arrhythmia; GVHD, graft-versus-host disease; COPD, chronic obstructive pulmonary disease; RAP; right atrial pressure; RVSP, right ventricular systolic pressure; PAP, pulmonary artery pressure; PCWP, pulmonary capillary wedge pressure; PCPS, percutaneous cardiopulmonary support; MV, mechanical ventilation; TnT, troponin T; ICU, intensive care unit. 
Table 2. Uni- and multivariate analyses for predictors of postoperative atrial arrhythmia

\begin{tabular}{|c|c|c|c|c|}
\hline \multirow{2}{*}{ Risk factor } & \multicolumn{2}{|c|}{ Univariate analysis } & \multicolumn{2}{|c|}{ Multivariate analysis } \\
\hline & OR $(95 \% \mathrm{CI})$ & $p$ value & OR $(95 \% \mathrm{CI})$ & $p$ value \\
\hline Age at transplant, /1 yr & $1.05(1.02-1.09)$ & 0.002 & $0.97(0.92-1.03)$ & 0.327 \\
\hline Body surface area, $/ 1 \mathrm{~m}^{2}$ & $8.35(1.10-63.59)$ & 0.040 & $3.09(0.11-84.47)$ & 0.504 \\
\hline History of atrial fibrillation & $10.44(1.13-96.18)$ & 0.038 & $5.24(0.39-71.11)$ & 0.213 \\
\hline Idiopathic pulmonary fibrosis & $2.41(1.13-5.17)$ & 0.024 & $2.30(0.62-8.63)$ & 0.216 \\
\hline RAP & $1.09(0.99-1.19)$ & 0.060 & $1.19(1.06-1.35)$ & 0.005 \\
\hline MV duration, / 1 day & $1.03(1.01-1.05)$ & 0.004 & $1.03(1.01-1.06)$ & 0.008 \\
\hline
\end{tabular}

OR, odds ratio; CI, confidence interval; RAP, right atrial pressure; MV, mechanical ventilation.

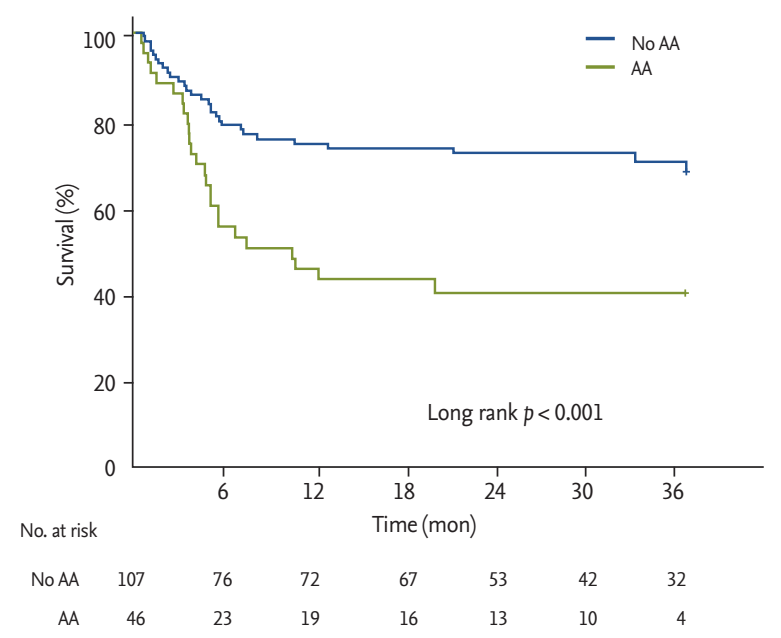

Figure 3. Kaplan-Meier curves for survival in patients with and without atrial arrhythmias after lung transplantation. AA, atrial arrhythmia.

Table 3. All-cause mortality and cause of death in patients with and without atrial arrhythmia

\begin{tabular}{lccc}
\hline Variable & AA $(\mathrm{n}=46)$ & $\begin{array}{c}\text { No AA } \\
(\mathrm{n}=107)\end{array}$ & p value \\
\hline 30-day mortality & $3(6.5)$ & $2(1.9)$ & 0.323 \\
90-day mortality & $8(13.0)$ & $11(10.3)$ & 0.780 \\
1-yr mortality & $23(50.0)$ & $25(23.4)$ & 0.001 \\
All-cause mortality & $25(54.3)$ & $29(27.1)$ & 0.001 \\
Cause of death & & & \\
\multicolumn{1}{l}{ Sepsis } & $16(64.0)$ & $18(62.1)$ & \\
Bleeding & $3(12.0)$ & $4(13.8)$ & \\
Cardiac death & $2(8.0)$ & $2(6.9)$ & \\
Multi-organ failure & $4(16.0)$ & $4(13.8)$ & \\
Unknown & 0 & $1(3.4)$ & \\
\hline
\end{tabular}

Values are presented as number (\%).

AA, atrial arrhythmia.

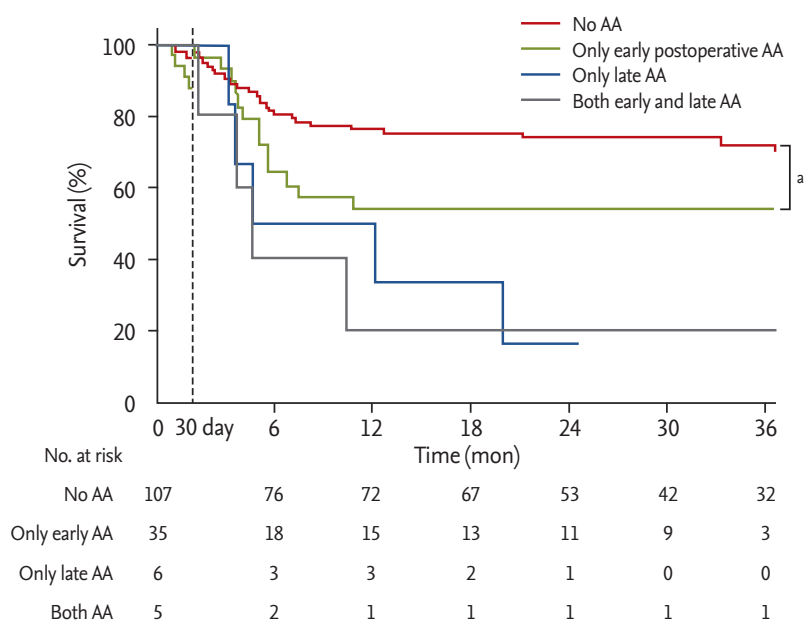

Figure 4. Landmark analysis for survival after the first 30 days post transplantation. AA, atrial arrhythmia. ${ }^{a}$ Log lank $p=0.001$.

mortality after LT was higher in patients with AA than in those without AA.

\section{Potential mechanisms of AA after LT}

Our data shows that AA occurred most frequently in the early postoperative period (within 30 days) and its frequency declined after 1 month. AF was the most commonly occurring AA in the early postoperative period, whereas late AA was composed of mainly AT and AFL. These observations are consistent with previously reported data $[4,5,14]$. AF could be occurring frequently during the early postoperative period due to local inflammation, myocardial injury, sympathetic activation, fluid shift, and electrolyte imbalances [15-18]. Additionally, the operative technique during LT, such as sutured anastomosis between the donor's pulmonary 
Table 4. Predictors for overall mortality after lung transplantation

\begin{tabular}{|c|c|c|c|c|}
\hline \multirow{2}{*}{ Risk factor } & \multicolumn{2}{|c|}{ Univariate analysis } & \multicolumn{2}{|c|}{ Multivariate analysis } \\
\hline & $\mathrm{HR}(95 \% \mathrm{CI})$ & $p$ value & $\mathrm{HR}(95 \% \mathrm{CI})$ & $p$ value \\
\hline Age at transplant, /1 yr & $1.02(1.00-1.04)$ & 0.071 & $1.00(0.97-1.04)$ & 0.894 \\
\hline Male sex & $1.64(0.93-2.89)$ & 0.088 & $1.82(0.75-4.39)$ & 0.186 \\
\hline History of HTN & $1.93(1.08-3.43)$ & 0.026 & $1.13(0.46-2.77)$ & 0.788 \\
\hline History of AF & $7.17(0.78-65.81)$ & 0.082 & $0.79(0.36-3.38)$ & 0.646 \\
\hline $\mathrm{E} / \mathrm{E}^{\prime}>15$ & $3.01(1.44-6.31)$ & 0.003 & $4.43(1.55-12.70)$ & 0.006 \\
\hline Preoperative PCPS & $2.69(1.51-4.81)$ & 0.001 & $2.01(0.49-8.20)$ & 0.330 \\
\hline Preoperative mechanical ventilation & $2.98(1.73-5.14)$ & $<0.001$ & $1.09(0.35-3.43)$ & 0.883 \\
\hline MV duration, / 1 day & $1.04(1.03-1.05)$ & $<0.001$ & $1.03(1.01-1.05)$ & $<0.001$ \\
\hline Atrial arrhythmia after LT & $2.49(1.45-4.26)$ & 0.001 & $2.75(1.20-6.32)$ & 0.017 \\
\hline
\end{tabular}

HR, hazard ratio; CI, confidence interval; HTN, hypertension; AF, atrial fibrillation; PCPS, percutaneous cardiopulmonary support; MV, mechanical ventilation; LT, lung transplantation.

vein cuffs and the recipient's left atrium, can provide an electroanatomic substrate for potential AA $[4,19]$. However, it could also serve as an antral electrical isolation of the pulmonary veins since the suture line is similar with the line ablation created by the Cox-Maze operation and can theoretically provide an antiarrhythmogenic effect for AF in the long-term. Later occurrences of AFL or AT than AF after LT could be due to reentry from the anastomosis after healing. Azadani et al. [14] reported a greater proportion of atypical pattern of AFL in recipients who underwent LT and proposed that atypical AFL stems from the suture line of the left atrium in contrast to typical AFL, which arises from the right atrium around the tricuspid annulus. In an electrophysiological study, See et al. [4] showed cases of postoperative AT related to macro-reentry that originated from suture lines, and focal AT that arose from the pulmonary vein anastomoses. Taken together, these results suggest that surgical suture lines can help prevent AF, and can also act as substrate for the development of AFL or AT after scarring. Additional electrophysiological studies must be carried out to confirm this theory and better understand the mechanism of AA after LT.

\section{Risk factors for AA after LT}

In this study, we identified that elevated RAP and a prolonged period of mechanical ventilation period were independent predictors of AA, after adjusting for confounding variables. Mechanical ventilation in- duces intermittent positive pressure during respiratory cycles, which may raise intrathoracic pressure and right atrial pressure. Regardless, due to the application of additional positive end expiratory pressure, right atrial pressure may remain higher throughout the respiratory cycle [20]. Such elevated right atrial pressure is related with occurrences of AA. Furthermore, prolonged periods of mechanical ventilation can cause an increase in sympathetic activity, which may trigger the development of AA.

\section{Clinical significance of AA after LT}

Long-term all-cause mortality was significantly higher in patients with AA. Furthermore, the development of AA was identified as an independent predictor of overall mortality in the multivariate analysis. Interestingly, even though most AA occurred in the early postoperative period, early clinical outcomes, such as length of ICU stay, length of hospital stay, and in-hospital mortality, were not different between patients with and without AA in our study population. However, we clarified the prognostic impact of early postoperative AA on survival clearly in the long term via our landmark analysis. These findings are in agreement with the results reported by Orrego et al. [3], which demonstrated a higher 12-month overall mortality in those who developed AA, but not at 90 days. Despite conflicting results of the impact of postoperative AA on long term survival after LT [11,21], a recent meta-analysis demonstrated that the occurrence of AA after LT has prognostic implications 
for overall long-term survival [22]. Taken together, these results suggest that development of AA after LT may not influence short-term mortality, but likely affects overall long-term mortality. Moreover, we found that development of late AA was also linked with poor long-term survival after LT. To our knowledge, an association between late AA after LT and survival has not been reported in prior studies. In this study, we could not identify the differences in characteristics between patients with late AA and their counterparts due to a small sample size. Further large-scale studies need to be carried out to address this issue.

The mechanisms that drive postoperative AA and result in poor long-term survival are not known. Mean time to death after development of AA was $76.0 \pm 78.5$ days in our study. Most patients died from infection, bleeding or multi-organ failure, and these poor medical conditions could have acted as a trigger for AA. In addition, longer periods of perioperative mechanical ventilation, which was related to development of AA, may reflect the more complex surgery and complications like infection. Therefore, we speculate that AA may serve as a potential surrogate marker to identify unstable medical conditions that may make patients vulnerable to stress and may increase their risk of mortality.

\section{Study limitations}

There are several limitations to be noted. Given the retrospective nature of this study at a single institution with a small number of patients, unmeasured confounders associated with AA such as consistent surgical techniques or postoperative care might have biased the results despite statistical adjustments. Further, some short and asymptomatic arrhythmic episodes during inconsistent ECG monitoring in the general ward or late AA events could have been missed. However, given complete access and review of these events, it is not likely that meaningful information was lost. Additionally, we could not determine the mechanisms driving postoperative AA due to unavailable electrophysiological data in our study. Further large-scale electrophysiological studies are required to better understand the mechanisms of AA and establish prevention strategies in $\mathrm{LT}$ recipients.

In conclusion, patients with preoperative RAP and long-term ventilator care had a higher risk of developing AA after LT. Further, AA after LT is associated with poor long-term survival.

\section{KEY MESSAGE}

1. Atrial arrhythmia (AA) is common in patients who underwent lung transplantation (LT).

2. Elevated preoperative right atrial pressure and long-term ventilator care were associated with development of AA after LT.

3. AA after LT is linked with poor long-term survival.

\section{Conflict of interest}

No potential conflict of interest relevant to this article was reported.

\section{REFERENCES}

1. Yusen RD, Christie JD, Edwards LB, et al. The Registry of the International Society for Heart and Lung Transplantation: thirtieth adult lung and heart-lung transplant report. 2013. Focus theme: age. J Heart Lung Transplant 2013;32:965-978.

2. Christie JD, Edwards LB, Aurora P, et al. Registry of the International Society for Heart and Lung Transplantation: twenty-fifth official adult lung and heart/lung transplantation report. 2008. J Heart Lung Transplant 2008;27:957-969.

3. Orrego CM, Cordero-Reyes AM, Estep JD, et al. Atrial arrhythmias after lung transplant: underlying mechanisms, risk factors, and prognosis. J Heart Lung Transplant 2014;33:734-740.

4. See VY, Roberts-Thomson KC, Stevenson WG, Camp PC, Koplan BA. Atrial arrhythmias after lung transplantation: epidemiology, mechanisms at electrophysiology study, and outcomes. Circ Arrhythm Electrophysiol 2009;2:504510.

5. Chaikriangkrai K, Jyothula S, Jhun HY, et al. Incidence, risk factors, prognosis, and electrophysiological mechanisms of atrial arrhythmias after lung transplantation. JACC Clin Electrophysiol 2015;1:296-305.

6. Isiadinso I, Meshkov AB, Gaughan J, et al. Atrial arrhyth- 
mias after lung and heart-lung transplant: effects on short-term mortality and the influence of amiodarone. J Heart Lung Transplant 2011;30:37-44.

7. D'Angelo AM, Chan EG, Hayanga JW, et al. Atrial arrhythmias after lung transplantation: incidence and risk factors in 652 lung transplant recipients. J Thorac Cardiovasc Surg 2016;152:901-909.

8. Nielsen TD, Bahnson T, Davis RD, Palmer SM. Atrial fibrillation after pulmonary transplant. Chest 2004;126:496-500.

9. Mason DP, Marsh DH, Alster JM, et al. Atrial fibrillation after lung transplantation: timing, risk factors, and treatment. Ann Thorac Surg 2007;84:1878-1884.

10. Malik A, Hsu JC, Hoopes C, Itinarelli G, Marcus GM. Elevated pulmonary artery systolic pressures are associated with a lower risk of atrial fibrillation following lung transplantation. J Electrocardiol 2013;46:38-42.

11. Henri C, Giraldeau G, Dorais M, et al. Atrial fibrillation after pulmonary transplantation: incidence, impact on mortality, treatment effectiveness, and risk factors. Circ Arrhythm Electrophysiol 2012;5:61-67.

12. Eberlein M, Garrity ER, Orens JB. Lung allocation in the United States. Clin Chest Med 2011;32:213-222.

13. Uhm JS, Park MS, Joung B, Pak HN, Paik HC, Lee MH. Intra-atrial reentrant tachycardia originating from the pulmonary vein cuff anastomosis in a lung transplantation patient: ultra-high-density 3-dimensional mapping. HeartRhythm Case Rep 2018;4:152-154.

14. Azadani PN, Kumar UN, Yang Y, et al. Frequency of atrial flutter after adult lung transplantation. Am J Cardiol
2011;107:922-926.

15. Echahidi N, Pibarot P, O'Hara G, Mathieu P. Mechanisms, prevention, and treatment of atrial fibrillation after cardiac surgery. J Am Coll Cardiol 2008;51:793-801.

16. Bazaz R, Salizzoni S, Bonde P, Espinoza A, Toyoda Y. A novel strategy for prevention of post-operative atrial arrhythmias in patients undergoing lung transplantation. J Heart Lung Transplant 2010;29:713-715.

17. Maesen B, Nijs J, Maessen J, Allessie M, Schotten U. Post-operative atrial fibrillation: a maze of mechanisms. Europace 2012;14:159-174.

18. Shingu Y, Kubota S, Wakasa S, Ooka T, Tachibana T, Matsui Y. Postoperative atrial fibrillation: mechanism, prevention, and future perspective. Surg Today 2012;42:819824 .

19. Dizon JM, Chen K, Bacchetta M, et al. A comparison of atrial arrhythmias after heart or double-lung transplantation at a single center: insights into the mechanism of post-operative atrial fibrillation. J Am Coll Cardiol 2009;54:2043-2048.

20. Shekerdemian L, Bohn D. Cardiovascular effects of mechanical ventilation. Arch Dis Child 1999;80:475-480.

21. Jesel L, Barraud J, Lim HS, et al. Early and late atrial arrhythmias after lung transplantation: incidence, predictive factors and impact on mortality. Circ J 2017;81:660-667.

22. Fan J, Zhou K, Li S, Du H, Che G. Incidence, risk factors and prognosis of postoperative atrial arrhythmias after lung transplantation: a systematic review and meta-analysis. Interact Cardiovasc Thorac Surg 2016;23:790-799. 